\title{
An Intelligent Service System for Outdoor Cycling
}

\author{
Huiyan Wang and Jialiang $\mathrm{He}^{*}$ \\ College of Information and Communication Engineering, Dalian Nationalities University, China \\ ${ }^{*}$ Corresponding author
}

\begin{abstract}
This product aims to make sure that the rider concentrate on the road. Therefore, we adopted an intelligent hardware+APP+Data platform. Its unique feature is that low-cost and simple electronic equipment which has the same function, not only flexible system design, but also LED lights as a navigation for user interface, solving the trouble of parking to look at the map when cycling.
\end{abstract}

\section{Keywords-sensor; intelligent service system}

\section{INTRODUCTION}

LeTu has been used in many domains of traffic and logistics[1-5]. As more and more people around us who choose to ride and other outdoor sports as a leisure adjustment and fitness activity after work, people demand for outdoor sports derived related equipment is bigger and bigger. It will be a broad market. Nowadays, smart bikes and intelligent accessories of bikes are multifarious, some equip with the displays which are power-consuming for the user to see all kinds of sports consumption, data of bikes' status and a map with full details, but for riding people, the most important thing of road riding is safety, rather than a fancy and inefficient user interface. As an intelligent bicycle equipment, the design of LeTu aims to make sure that the rider concentrate on the road.
Mobile phone as an intelligent equipment with a lot of smart devices, the function is not only strong but also free, but when it is used in the ride scene, it has many trouble, such as three proofing ability, endurance ability, the visibility of the sunlight under the screen, the convenience of installation and so on, so it needs specialized intelligent hardware equipment. The integration of the solution which is intelligent hardware + APP + Data platform, for users, they will enjoy a better and more complete experience. Therefore, we have designed a low-cost and simple electronic equipment which has the same function, not only flexible system design, but also LED lights as a navigation for user interface, solving the trouble of parking to look at the map when cycling. The basic function can be used as an outdoor sports environment parameter tester. It can realize temperature, humidity and PM2.5 real-time collecting data processing platform, route planning and navigation.

\section{SySTEM DESIGN}

System consists of GPS, temperature sensor, ambient light sensor and other sensor modules, LCD display module, such as EEPROM storage module, RTC module, bluetooth 4.0 communication module and lithium battery modules.

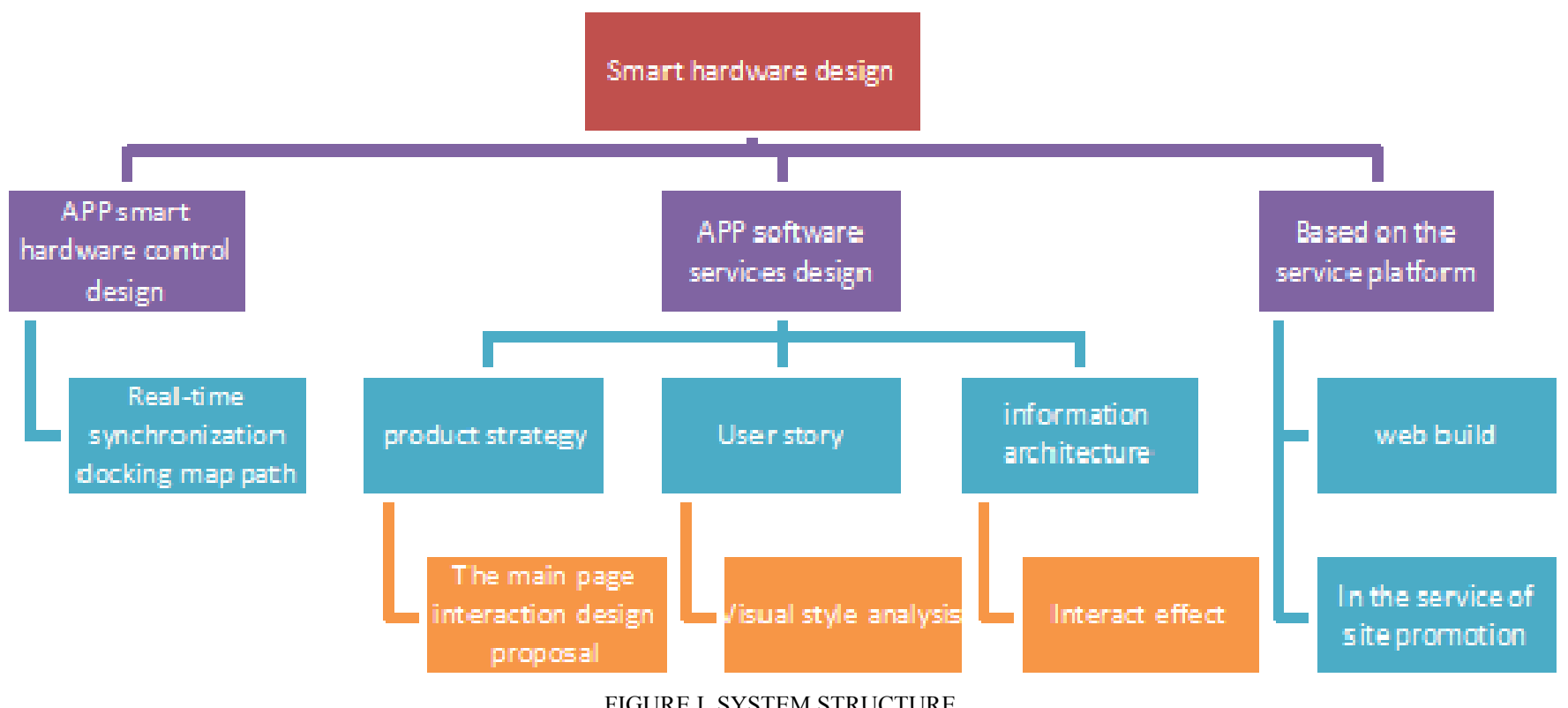




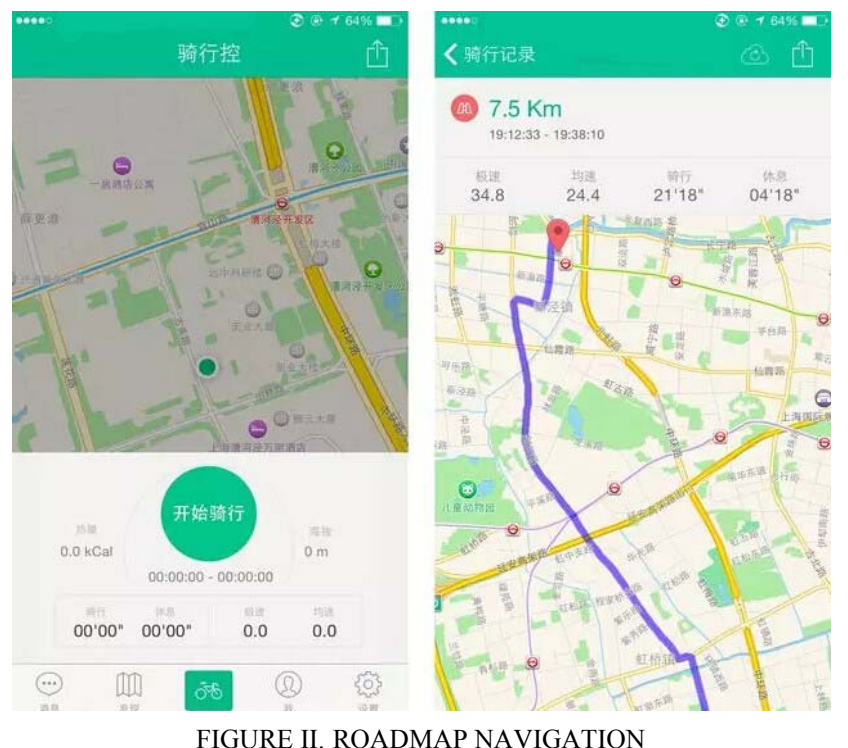

Hardware aspects: single chip microcomputer control. processing of each sensor measurement information and the backlight electronic paper screen real-time display; EEPROM storage path information; LeTu built-in magnetometer, accelerometer, gyroscope, encryption, authentication, bluetooth 4.0 low power consumption and low power consumption LED lights, nocturnal car lamp brightness of 250 lumens, battery capacity is $2000 \mathrm{ma}$, USB port at the same time have the effect of lithium batteries, enable strong battery life. Power management of the peripheral module, adopt the method of minimum system with MCU power supply separately, so you can shut down at any time temporarily without sensor, to save electricity. Positioning handheld devices, batteries, selection of devices are low-power devices.

Software aspects: LeTu supports with IOS apple smartphones and bluetooth 4.0 Android smartphones, users can upgrade the firmware via bluetooth communication way. Backlit electronic paper screen.

The whole system consists of several modules, system block diagram as shown:

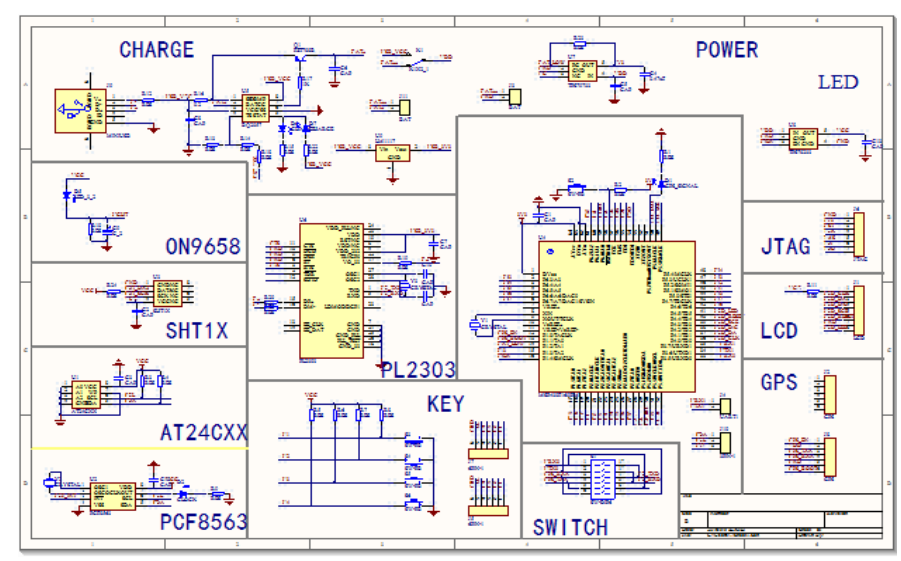

FIGURE III. THE PRINCIPLE DIAGRAM OF THE HARDWARE

\section{The Design OF Systematic HARDWARE}

\section{A. Modules of Power Supply/Charge}

The system has two approaches of power supply: USB power supply and lithium battery, In peacetime, lithium battery is utilized for power supply, When connecting with upper computer, we generally utilize USB power supply. It could also provide power for lithium battery at the same time. On account of low power consumption, the choice of chip is crucial, consequently we choose the chip of minimum quiescent current and low power consumption.

The design of system requires simple compact peripheral circuit.And the recharger possess Auto Recharging, temperature measurement, three-stage charging function etc. Integrating these requires, we choose the Lithium-Ion Linear Battery Charge Controller of Texas Instruments.

\section{B. GPS Module}

The real-time display contains the state of the GPS, signal strength, longitude and latitude, height and speed, time and date etc. By using button, we can realize run or cease of GPS, recordation or delete of Coordinate data, the record of manual /Automatic switch, upper computer connecting etc. EEPROM AT24C64 is utilized for recording Coordinate information.

One information contains 64 bytes and storages 512 recordings.

On the basis of 1 piece of information per min, it can have 8 hours working, The recordation of information can not only be recorded by manual button. but can be switched to $10 \mathrm{~s} / 30 \mathrm{~s} / 60 \mathrm{~s} / 90 \mathrm{~s}$ interval's Automatic recording.

Data which is recording in EEPROM transfer to a PC through a serial port, PC software convert data, also can be directly output KML files, open the drawing path in computer software. Our company is trying to combine the Android to realize remote data acquisition and real-time synchronous record path.

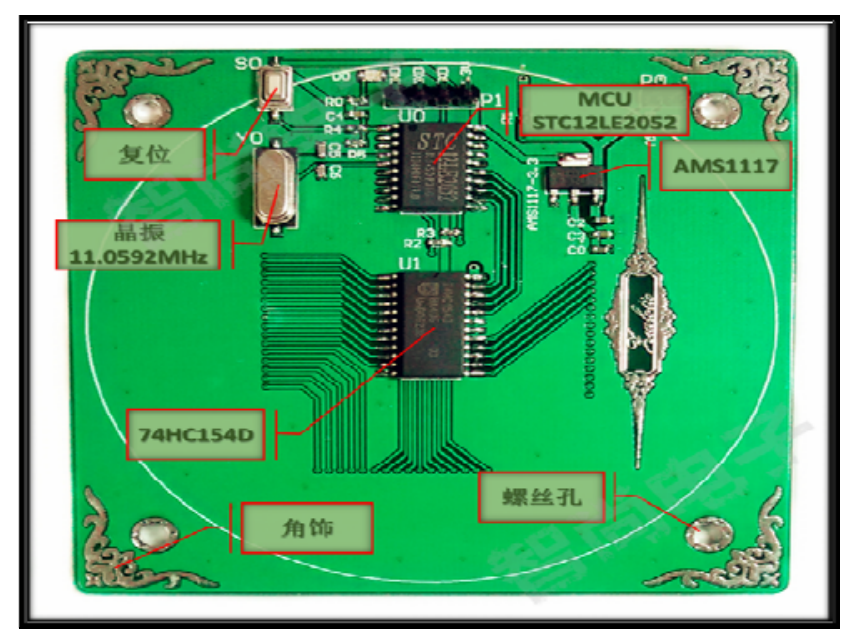

FIGURE IV. PRODUCT COMMUNICATION MODULE

\section{Communication Module}

When communicating with PC, MCU uploads coordinate data. The main function of upper computer is transform the 
recorded data, then generate KML file which can be opened directly. When GPS communicates with PC, PC software displays decoding information and $\mathrm{MCU}$ then communicates with GPS, LCD display decoding information.

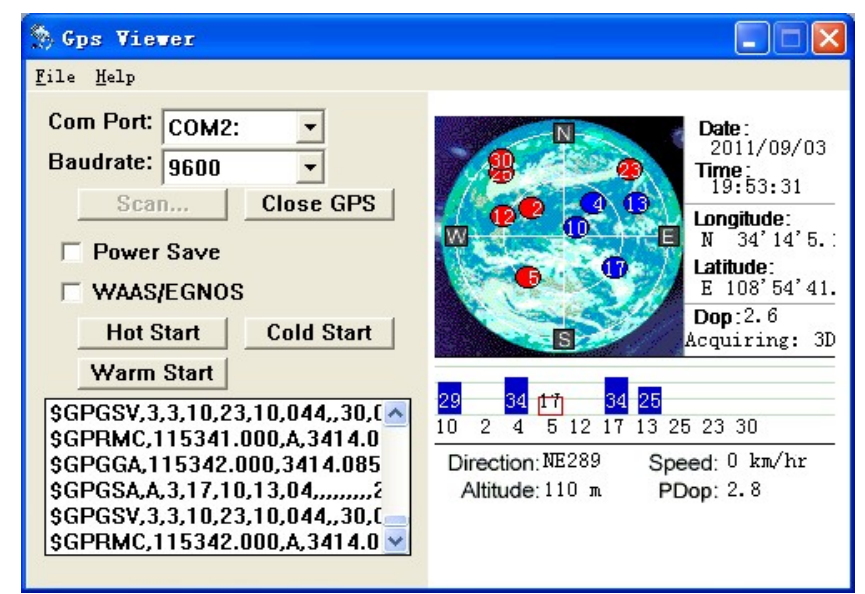

FIGURE V. PRODUCT COMMUNICATION MODULE SOFTWARE

PART

\section{Sensor and Related Modules, Display Module}

Electronic display screen adopts partly refresh. This way the screen only need to refresh changing are, consequently it saves resources. Showing simulation time needs wide range of refresh, in order to avoid LCD blinking and display dislocation when directly manipulating. The program open up display buffer area.Analog clock pointer is manipulated in display buffering area. After finishing operation, LCD directly display the data of display buffer area, regarding ascorresponding UI.

Add sensors, such as Light sensor, speed sensor etc. The typical incident wavelength of light is $\lambda p=520 \mathrm{~nm}$, built-in double sensitive receiver, the visible range is highly sensitive andthe output current varies linearly with the illumination intensity. These are suitable forenergy saving control, adaptive regulation of automatic sensitization $\mathrm{TV}$, LCD backlight, electronics instruments and apparatuses etc.

\section{ACKNOWLEDGEMENT}

This work was supported by "National Undergraduate Training Programs for Innovation and Entrepreneurship of Dalian Nationalities University".

\section{REFERENCES}

[1] Feng Haiyang, He Jialiang, Zhang Lijun, Meng Fangang, An Application of RFID Technology in Traffic Congestion Management[C] Applied Mechanics and Materials, 2014, vol.513-517, pp.3954-3957.

[2] Shen Peng, He Jialiang, Feng Haiyang, A Solution of Vehicle Emission Inspection Using RFID Technology[C]. 2014 International Conference on Vehicle, Mechatronics and Information Technologies II. Applied Mechanics and Materials, 2014, vols.543-547, pp.946- 949

[3] Wang Huan, He Jialiang, Pan Yu. A Solution Framework in Traffic Congestion Management Using RFID Technology[C]. International Industrial Informatics and Computer Engineering Conference (IIICEC 2015). 2015, pp.1098-1101. Atlantis Press.

[4] He Jialiang, Shen Peng, Xu Zhiqiang. Inspection Solution of Unlawfully Modified Vehicle by Sensor-RFID Technology[J]. Sensors and Transducers Journal. 2013, vol.25, pp.197-202.
[5] He Jialiang, Xu Zhiqiang. Authentication and Search Mechanism for Diffusing RFID-Sensor Networks[J]. International Journal of Sensor Networks. 2013, vol.14, no.4, pp. 211-217. 